\title{
Cosmological models coupled with dark matter in a dissipative universe
}

\author{
I. Brevik • V. V. Obukhov • A. V. Timoshkin
}

January 13, 2021

\section{Abstract}

We consider the cosmological system with two interacting fluids: dark energy and dark matter, in a homogeneous and isotropic universe with dissipation. The modified gravitational equation for dark matter is solved. The analytic representations for the Little Rip, the Pseudo Rip, and the bounce cosmology models with dissipation are obtained in terms of the thermodynamic parameters in the equation of state. We analyze the corrections in the energy density for dark matter, in view of the dissipative processes and the coupling with dark energy.

Keywords dark energy, dark matter, viscous cosmology

\section{Introduction}

According to the modern cosmological picture the universe is filled with a negative pressure fluid (dark energy) which accounts for about $73 \%$ of the total mass/energy of the universe and only $27 \%$ of a combination of dark matter and baryonic matter; cf. Riess et al.

\section{Brevik}

Department of Energy and Process Engineering, Norwegian University of Science and Technology, N-7491 Trondheim, Norway

V. V. Obukhov

Tomsk State Pedagogical University, 634061 Tomsk, Russia, National Research Tomsk State University, Lenin Avenue, 36, 634050 Tomsk, Russia

\section{A. V. Timoshkin}

Tomsk State Pedagogical University, 634061 Tomsk, Russia, National Research Tomsk State University, Lenin Avenue, 36, 634050 Tomsk, Russia

${ }^{1}$ Affilation

(1998); Perlmutter et al. (1999). One phenomenological approach to describe the dark energy is to assume that this fluid obeys an equation of state with constant thermodynamic parameter $w=p / \rho$, where $\rho$ is the dark energy density and $p$ is the dark pressure; cf. Li et al. (2011); Bamba et al. (2012); Nojiri and Odintsov (2007). It is known from observational data that in the current universe the value of $w$ lies near, or probably below, $-1, w=-1.04_{-0.10}^{+0.09}$; cf. Kowalski (2008). The region where $w<-1$ is called the phantom region.

Various cosmological models for the evolution of the universe have been presented. They aim at explaining the acceleration era of the universe, and they include future singularity phenomena such as the dramatic Big Rip Caldwell et al. (2003); Nojiri and Odintsov [2004, 2003)], the softer Little Rip [Frampton et al. (2011); Brevik et al. (2011); Frampton et al. (2012)], the Pseudo Rip [Frampton et al. (2012a)], the Quasi Rip Wei et al. (2012)], and the bounce cosmology Brandenberger (2011); Novello and Bergliatta (2008); Bamba (2013); Cai et al. (2011); Brevik and Timoshkin (2015)]. The Little Rip and the Pseudo Rip phenomena, and the bounce cosmology pictured as an inhomogeneous nonviscous dark fluid coupled with dark matter, were considered by Brevik et al. (2013, 2014). Cosmological models in which the modification of gravity is considered as a viscous fluid are explored by Mvrzakul et al. (2013); Mvrzakulov and Sebastiani (2014); Elizalde et al. (2014). Investigations dealing with the coupling between the dark energy and dark matter components are considered by Brevik et al. (2014); Timoshkin (2009). A very extensive review on cosmological evolution with interaction between dark energy and dark matter in general is recently given by Bolotin et al. (2015).

A main motivation for the present study is to investigate the influence from heat dissipation. We will thus 
study the Little Rip, the Pseudo Rip, and the bounce cosmology, by considering a homogeneous dark fluid interacting with dark matter. This means that each fluid component constitutes a non-closed physical system, implying exchange of energy with its companion fluid component. Mathematically, it means the appearance of a source term on the right hand side of the energy conservation equation; cf. Eq. (11) below. We will assume that for each fluid component (dark energy and dark matter) there exists a linear and homogeneous equation of state; this being the simplest alternative. We moreover assume a spatially flat, homogeneous and isotropic FRW space.

Analytical expressions will be worked out for the cosmological cases mentioned above, and we will make appropriate choices for the various thermodynamical parameters. Energy dissipation is conveniently expressed in terms of the bulk viscosity, as this kind of viscosity is compatible with the assumption about spatial isotropy. It should be mentioned, however, that this is actually a delicate point. The shear viscosity calculated in cosmology on the basis of the Boltzmann equation (cf. Hogeveen et al. (1986)) is much larger than the bulk viscosity. Thus even a minute anisotropy in the universe might make the shear viscosity an important component. The shear viscosity will however be omitted here, in accordance with common usage.

Our exposition will be related to the so-called entropic force model - cf. Eq. (4) below - in which the equation of state has quite a general form, expressed in terms of powers of the Hubble parameter.

Finally, as a general point one may ask: what are the physical reasons for introducing viscosity coefficients in the cosmic fluid? This question can be considered from different angles. First, from a hydrodynamical point of view it is apparent that the ideal (nonviscous) fluid model applies only under restricted circumstances. Once there are irreversible process involved, the introduction of one or two viscosity coefficients becomes inevitable. Second, in a cosmological context the inclusion of viscosity broadens the applicability of the theory considerably. Not least so, this applies in connection with the transition to turbulent motion which is quite probable when the fluid approaches the future singularity Brevik et al. (2011)]. Even in the very early universe, in connection with the inflationary period, viscosity has been proposed in order to deal with the grateful exit; cf. Myrzakulov and Sebastiani (2014a). It is thus quite understandable that a large number of papers on viscosity has appeared on viscous cosmology. Readers interested in review papers may consult, for instance, Grøn (1990); Brevik and Grøn (2013).

\section{Dissipative cosmologies coupled with dark matter}

We consider a phenomenological model in which there occurs a constant entropic pressure associated with the dark matter fluid component. This falls into line with the so-called entropic cosmology model for a dissipative universe (see the paper by Komatsu and Kimura (2014) and further references therein). This is a generalized form of cosmology combining bulk viscosity theory with creation theory of cold dark matter.

The modified continuity and acceleration equations for the interacting fluid system can be written as

$$
\left\{\begin{array}{l}
\dot{\rho}+3 H(\rho+p)=-\tilde{Q} \\
\dot{\rho}_{m}+3 H\left(\rho_{m}+p_{e}\right)=\tilde{Q} \\
\dot{H}=-\frac{k^{2}}{2}\left(\rho+p+\rho_{m}+p_{e}\right)
\end{array}\right.
$$

Here $H=\dot{a} / a$ is the Hubble parameter, and $k^{2}=8 \pi G$ with Newton's gravitational constant $G$. Moreover $\rho, p$ and $\rho_{m}, p_{m}$ are the energy density and pressure respectively of dark energy and dark matter, and $\tilde{Q}$ describes the interaction. A dot means derivative with respect to cosmic time $t$. We put $c=1$. Quantities referring to present time $t=0$ are given a subscript zero.

The first line in Eq. (1) is the continuity equation for dark energy [Nojiri et al. (2005a)]. The second line is the continuity equation for dark matter, in which $p_{e}$ is the mentioned constant pressure term. This term describes the influence from dissipation, and is connected with the temperature and the entropy of the Hubble horizon. It describes irreversible entropy, generated either by a bulk viscosity in one formulation, or with the creation of cold dark matter in the alternative formulation (a closer discussion on this point can be found in Komatsu and Kimura (2014) and earlier papers). We assume that the dark matter is dust, so that its mechanical pressure is $p_{m}=0$.

The constant effective pressure $p_{e}$ has to be a negative quantity. Compare here with the behavior of viscous cosmology where the thermodynamic pressure $p$ is reduced by an amount $3 \zeta H$, where $\zeta$ is the bulk viscosity, necessarily positive from thermodynamic grounds. We shall write it as $p_{e} \propto-2 H_{0}^{2} / k^{2}$, with a positive constant of proportionality. Calling this constant $(\mu-\gamma)$ (because of the use of $\gamma$ in the subsequent Friedmann equation (3) $)$, we must have $\mu \geq \gamma \geq 0$. Thus

$p_{e}=-\frac{2 H_{0}^{2}}{k^{2}}(\mu-\gamma)$,

and the Friedmann equation is written as

$H^{2}=\frac{k^{2}}{3}\left(\rho+\rho_{m}\right)+\gamma H_{0}^{2}$ 
The additional term $\gamma H_{0}^{2}$ corresponds to an entropicforce term derived from reversible entropy in the entropic cosmology model; cf. Komatsu and Kimura (2014). The term can be interpreted as an effective dark energy term. The constant effective pressure $p_{e}$ in Eq. (2) consists of contributions from the entropy term to the pressure. They turn up in the coefficients $w_{i}(t)$ given in Eq. (4) below.

We consider the following general equation of state for the dark energy Capozziello et al. (2005); Noiiri and a (2006)]

$p=w_{4}(t) H^{4}+w_{3}(t) H^{3}+w_{2}(t) H^{2}+w_{1}(t) H$,

where the thermodynamic parameters $w_{1}, w_{2}, w_{3}, w_{4}$ are so far undetermined functions of the cosmic time $t$. Higher order terms are neglected because inflation in the early universe is not considered.

We turn now to dissipative properties of the interacting dark energy universe, via proper adjustments of the parameters in Eq. (4).

\subsection{Little Rip model}

Let us assume the Little Rip model where the Hubble parameter increases exponentially with $t$,

$H=H_{0} e^{\lambda t}, \quad H_{0}>0, \lambda>0$

(recall that $t=0$ is present time).

Consider again the continuity equation for dark matter

$\dot{\rho}_{m}+3 H\left(\rho_{m}+p_{e}\right)=\tilde{Q}$,

and insert the following expression for the interaction term

$\tilde{Q}=\frac{H}{\lambda} H_{0}^{5}$.

In this case the solution of Eq. (6) for the dark matter is given by

$\rho_{m}(t)=\left(\rho_{0}-\Delta \rho_{m}\right) \exp \left(-\frac{3\left(H-H_{0}\right)}{\lambda}\right)+\Delta \rho_{m}$,

where $\rho_{m}(0)=\rho_{0}$ and the constant correction term $\Delta \rho_{m}$ is

$\Delta \rho_{m}=\frac{H_{0}^{5}}{\lambda}-p_{e}$.

From Eq. (8) one sees that $\rho_{m}(t \rightarrow \infty)=\Delta \rho_{m}$. This correction is caused by the exchange of energy between dark fluid and dark matter and by dissipative properties in the universe.
We now start from the gravitational equation of motion

$\frac{2 \lambda H}{k^{2}}+\rho+p+\rho_{m}+p_{e}=0$.

Choosing for the parameter $\gamma$ the value

$\gamma=\frac{p_{e}}{3}\left(\frac{k}{H_{0}}\right)^{2}$,

and choosing for the parameter $w_{1}(t)$ in the equation of state (4) the value

$w_{1}(t)=-\frac{2 \lambda}{k^{2}}$,

we can rewrite Eq. (10) in the simple form

$w_{4} H^{2}+w_{3} H+\left(w_{2}+\frac{3}{k^{2}}\right)=0$.

Thus, if we require that the parameters $w_{2}, w_{3}, w_{4}$ are related via the condition

$w_{3}^{2}-4 w_{4}\left(w_{2}+\frac{3}{k^{2}}\right)=0$,

we can from Eq. (13) determine the thermodynamic parameters as

$\left\{\begin{array}{l}w_{3}(t)=-2 H_{0} w_{4}(t) \exp (\lambda t), \\ w_{2}(t)=w_{4}(t) H_{0}^{2} \exp (2 \lambda t)-3 / k^{2}, \\ w_{4}(t) \in R .\end{array}\right.$

Insertion of these values in the equation of state (4) shows that the dark fluid pressure $p$ acquires an exponentially increasing behavior when $t \rightarrow \infty$. Thus the values for the w's chosen in Eqs. (12) and (15) lead to the characteristic features of the Little Rip cosmology. This circumstance motivates our parameter choices, including the ansatz (11) for $\gamma$. Physically, we have as a checking point that $\tilde{Q} \rightarrow 0$ when $t \rightarrow \infty$.

\subsection{Pseudo Rip model}

Now we will investigate a cosmological model where the dark energy is monotonically increasing. In this case the Hubble parameter asymptotically approaches a cosmological constant, which means that the universe approaches a de Sitter form.

We suppose that the Hubble parameter is given by Frampton et al. (2012)]

$H=H_{0}-H_{1} e^{-\tilde{\lambda} t}$,

where $H_{0}, H_{1}$ and $\tilde{\lambda}$ are positive constants, $H_{0}>H_{1}>$ $0, t>0$. 
Near the present time $(t \rightarrow 0)$ we have $H \rightarrow H_{0}-$ $H_{1}$, and in the late-time universe $(t \rightarrow \infty)$ the Hubble parameter tends to a constant.

We take now the coupling term to have the form

$\tilde{Q}=\delta H\left(\rho_{m}+p_{e}\right)$,

where $\delta$ is a positive nondimensional constant. This choice is motivated by the physically natural assumption that the coupling increases when the relative rate of change of the scale factor increases, i.e. when $H$ increases.

From Eq. (6) we find the energy density of dark matter

$\rho_{m}(t)=\left(\rho_{0}+p_{e}\right) \exp \left[(\delta-3)\left(H_{0} t-\frac{H-H_{0}+H_{1}}{\tilde{\lambda}}\right)\right]-p_{e}$

with $\rho_{m}(0)=\rho_{0}$ as before.

The gravitational equation of motion for dark energy becomes

$\frac{2 \tilde{\lambda}}{k^{2}}\left(H_{0}-H\right)+p+\frac{3}{k^{2}} H^{2}+p_{e}-\frac{3 \gamma}{k^{2}} H_{0}^{2}=0$,

with $\tilde{\lambda}=\frac{3}{2} \gamma H_{0}, \gamma$ being given by Eq. (11).

Thus, if we choose the thermodynamic parameters in the equation of state (4) to have the form

$$
\left\{\begin{array}{l}
w_{1}=\frac{3 \gamma}{k^{2}} H_{0} \\
w_{2}=-\frac{3}{k^{2}} \\
w_{3}(t)=\left[H_{1} \exp \left(-\frac{3}{2} \gamma H_{0} t\right)-H_{0}\right] w_{4}(t), \\
w_{4}(t) \in R
\end{array}\right.
$$

we obtain the characteristics of the Pseudo Rip dissipative universe. Assuming that $w_{4}$ stays finite when $t \rightarrow \infty$, all the other $w$ 's stay finite, and prevent the pressure in Eq. (4) from blowing up in the far future. Again, our choices of parameters are justified by the adjustment to one of the future singular cosmological models.

\subsection{Bounce cosmology}

In the matter bounce cosmology model, the universe goes from an era of accelerated collapse into an era of expansion via a bounce acting as a singularity. After the bounce, the universe is in a matter dominated phase.

Let us consider such a model where the scale factor $a$ has the exponential form [Bamba (2013)]

$a=\exp \left(\beta t^{2}\right)$ $\beta$ being a positive constant. The bounce occurs at $t=$ $0(a=1)$. We assume that $t \geq 0$ in the following. Now take the Hubble parameter to have the form

$H=2 \beta t$,

and take the interaction between dark energy and dark matter to be

$\tilde{Q}=Q_{0} \exp \left(-3 \beta t^{2}\right)$

with $Q_{0}=\tilde{Q}(0)$. Thus near the bounce $\tilde{Q} \rightarrow Q_{0}$, whereas for increasing values of $t, \tilde{Q} \rightarrow 0$ and the coupling becomes negligible.

Now the solution of Eq. (6) for the dark matter bepmes

$\mu_{m}(t)=\left(Q_{0} t+\rho_{0}+p_{e}\right) \exp \left(-3 \beta t^{2}\right)-p_{e}$,

with $\rho_{m}(0)=\rho_{0}$ as before. Just after the bounce the dark matter energy density thus increases linearly with $t$, whereas when $t \rightarrow \infty, \rho_{m} \rightarrow-p_{e}$. The correction $-p_{e}$ takes into account the dissipative processes.

We make the following choice for the parameter $\beta$,

$\beta=\frac{3}{4} \gamma H_{0}^{2}$

and write the gravitational equation for dark energy as

$\frac{4 \beta}{k^{2}}+\frac{3}{k^{2}}\left(H^{2}-\gamma H_{0}^{2}\right)+p+p_{e}=0$.

The conditions (4) and (25) lead to the simple form (26):

$w_{4} H^{4}+w_{3} H^{3}+\left(w_{2}+\frac{3}{k^{2}}\right) H^{2}+w_{1} H+p_{e}=0$.

From Eq. (26) one can find the following solution

$$
\left\{\begin{array}{l}
w_{1}(t)=-\frac{2}{3} \frac{p_{e}}{\gamma H_{0}^{2}} \frac{1}{t} \\
w_{2}=-\frac{3}{k^{2}} \\
w_{3}(t)=-\frac{3}{2} \gamma H_{0}^{2} w_{4}(t) t \\
w_{4}(t) \in R
\end{array}\right.
$$

It is seen that the singularity property at the bounce at $t=0$ is taken care of by the coefficient $w_{1}$.

We have thus in this subsection presented a description of dissipative bounce cosmology, related to the equation of state (4).

\section{Conclusion}

We have investigated some examples of Little Rip, Pseudo Rip, and the bounce cosmology, under conditions where a dark energy fluid is coupled with dark 
matter via an interaction term $\tilde{Q}$; cf. Eq. (1). The underlying geometry is a flat Friedmann-RobertsonWalker universe. The energy dissipation is most naturally described by means of a bulk viscosity, although there is a mathematically equivalent formalism implying creation of cold dark matter.

The formalism that we have made use of, falls within the so-called entropic force model Komatsu and Kimura $(2014)]$. We have assumed initially that the equation of state for the dark fluid has the general form (4), in which there occurs four thermodynamic coefficients $w_{i}, i \in[1,4]$. The various categories of singularitycontaining universes are then obtained by appropriate choices of these coefficients. Corrections to the dark matter energy density caused by dissipative processes are obtained.

Finally, we wish to make some additional comments on the physical meaning of the constant pressure $p_{e}$ introduced in Eq. (1), from the viewpoint of viscous cosmology. As mentioned above, the modification of thermodynamic pressure in a viscous fluid consists in replacing the thermodynamic pressure $p$ with $p-3 \zeta H$, with $\zeta$ the bulk viscosity. Thus, the quantity $p_{e}$ corresponds to $-3 H \zeta$, and the assumed constancy of $p_{e}$ implies that $\zeta$ has to be inversely proportional to $H$.

It is worth noticing that when this condition is satisfied, the Friedmann formalism for the viscous fluid becomes easily manageable. Let us recall from Brevik et al. (2011) how the formalism looks if one starts from the equation of state in the form

$p=-\rho-A \sqrt{\rho}+p_{e}$

for the dark fluid. Here $A$ is a constant, with dimension $\mathrm{cm}^{-2}$ in geometric units. In this case we find the following explicit formula for how $\rho$ depends on cosmic time $t(>0)$,

$t=\frac{2}{\sqrt{3} k} \frac{1}{A} \ln \frac{A \sqrt{\rho}-p_{e}}{A \sqrt{\rho_{0}}-p_{e}}$.

This can be inverted to give $\rho$ as a function of $t$,

$\rho(t)=\left[\left(\sqrt{\rho_{0}}-\frac{p_{e}}{A}\right) \exp \left(\frac{\sqrt{3}}{2} k A t\right)+\frac{p_{e}}{A}\right]^{2}$.

It is seen that the universe needs an infinite time to reach the state $\rho \rightarrow \infty$. This is just the characteristic property of Little Rip cosmology.

\section{Acknowledgments}

This work was supported by a grant from the Russian Ministry of Education and Science, project TSPU-139 (A.V.T. and V.V.O.). 


\section{References}

Bamba, K.; Capozziello, S.; Nojiri, S.; Odintsov, S. D.: Dark energy cosmology: the equivalent description via different theoretical models and cosmography tests. Astrophys. Space Sci. 342, 155 (2012), arXiv:1205.3421 [grqc].

Bamba, K.; Makarenko, A. N.; Myagky, A. N.; Nojiri, S.; Odintsov, S. D.: Bounce cosmology from $\mathrm{F}(\mathrm{R})$ gravity and $\mathrm{F}(\mathrm{R})$ bigravity, arXiv:1309.3748 1 [hep-th].

Bolotin, Yu.L.; Kostenko, A.; Lemets, O.A.; Yerokin, D.A.: Cosmological evolution with interaction between dark energy and dark matter. Int. J. Mod. Phys. D 24, 1530007 (2015), arXiv:1310.0085 v2 [astro-ph.CO].

Brandenberger, R. H.: Alternatives to cosmological inflation. Int. J. Mod. Phys. Conf. Ser. 01, 67 (2011), arXiv:0902.4731 [hep-th].

Brevik, I.; Timoshkin, A.: Inhomogeneous dark fluid and dark matter, leading to a bounce cosmology. Universe 1, 24 (2015), arXiv:1503.02916] [gr-qc].

Brevik, I.; Timoshkin, A. V.; Raboshaya, Y.: Little rip and pseudo rip phenomena from coupled dark energy. Mod. Phys. Lett. A 28, 1350172 (2013).

Brevik, I.; Obukhov, V. V.; Timoshkin, A. V.: Bounce universe induced by an inhomogeneous dark fluid coupled with dark matter. Mod. Phys. Lett. A 29, 1450078 (2014).

Brevik, I.; Elizalde, E.; Nojiri, S.; Odintsov, S. D.: Viscous little rip cosmology. Phys. Rev. D 84, 103508 (2011), arXiv:1107.4642 [hep-th].

Brevik, I.; Grøn, Ø.: Relativistic universe models. In: Recent Advances in Cosmology, p. 97. Nova Scientific Publishers, New York (2013).

Cai, Yi-Fu; Chen, Shin-Hung; Dent, J. B.; Dutta, S.; Saridakis, E. N.: Matter Bounce Cosmology with the f(T) Gravity. Class.Quantum Gravity 28, 215011 (2011), arXiv:1104.4349 [astro-ph.CO].

Caldwell, R. R.; Kamionkowski, M.; Weinberg, N. N.: Phantom energy and cosmic doomsday. Phys. Rev. Lett. 91, 071301 (2003), astro-ph/0302505.

Capozziello, S.; Cardone, V. F.; Elizalde, E.; Nojiri, S.; Odintsov, S. D.: Observational constraints on dark energy with generalized equation of state. Phys. Rev. D 73, 043512 (2006), hep-th/0508350

Elizalde, E.; Obukhov, V. V.; Timoshkin, A. V.: Inhomogeneous viscous dark fluid coupled with dark matter in the FRW universe. Mod. Phys. Lett. A 29, 1450132 (2014), arXiv:1406.7653 [gr-qc].

Frampton, H.; Ludwick, K. J.; Scherrer, R. J.: The little rip. Phys. Rev. D 84, 063003 (2011), arXiv: 1106.4996v1 [astro-ph. CO].

Frampton, P. H.; Ludwick, K. J.; Nojiri, S.; Odintsov, S. D.; Scherrer, R. J.: Models for little rip dark energy. Phys. Lett. B 708, 204 (2012), arXiv:1108.0067v2 [hep-th].

Frampton, P. H.; Ludwick, K. J.; Scherrer, R. J.: Pseudorip: Cosmological models intermediate between the cosmological constant and the little rip. Phys. Rev. D 85, 083001 (2012), arXiv:1112.2964 2 [astro-ph. CO].

Grøn, Ø.: Viscous inflationary universe models. Astrophys. Space Sci. 173, 191 (1990).

Hogeveen, F., van Leeuwen, W.A., Salvati, G.A.Q., Schelling, E.E.: Physica (Amsterdam) 134A, 458 (1986).
Komatsu, N.; and Kimura, S.: Entropic cosmology in a dissipative universe. Phys. Rev. D 90, 123516 (2014), arXiv:1408.4836. v1 [astro-ph. CO].

Kowalski, M.: Improved cosmological constraints from new, old and combined supernova datasets. Ap. J. 686, 749 (2008), arXiv:0804.4142 [astro-ph].

Li, M.; Li, X.; Wang, S.; Wang, Y.: Dark energy. Commun. Theor. Phys. 56, 525 (2011), arXiv:1103.5870 [astro-ph $\mathrm{CO}]$.

Myrzakul, S.; Myrzakulov, R.; Sebastiani, L.:(2013). Inhomogeneous viscous fluids in FRW universe and finitefuture time singularities. arXiv:1311.6939v1 [gr-qc].

Myrzakulov, R.; Sebastiani, L.:(2014). Bounce solutions in viscous fluid cosmology. arXiv:1403.0681 v1 [gr-qc].

Myrzakulov, R.; Sebastiani, L.:(2014a).Inhomogeneous viscous fluids for inflation. arXiv:1410.3573 2 [gr-qc].

Nojiri, S.; Odintsov, S. D.: Final state and thermodynamics of a dark energy universe. Phys. Rev. D 70, 1035222004.

Nojiri, S.; Odintsov, S. D.: Quantum de Sitter cosmology and phantom matter. Phys. Lett. B 562, 147 (2003).

Nojiri, S.; Odintsov, S. D.: Inhomogeneous equation of state of the universe: Phantom era, future singularity and crossing the phantom barrier. Phys. Rev. D 72, 023003 (2005), hep-th/0505215

Nojiri, S.; Odintsov, S. D.; Tsujikawa, S.: Properties of singularities in phantom dark energy universe. Phys. Rev. D 71, 063004 (2005), hep-th/0501025.

Nojiri, S.; Odintsov, S. D.: The new form of the equation of state for dark energy fluid and accelerating universe. Phys. Lett. B 639, 144 (2006), hep-th/0606025

Nojiri, S.; Odintsov, S. D.: Introduction to modified gravity and gravitational alternative for dark energy. Int. J. Geom. Meth. Mod. Phys. 4, 115 (2007), hep-th/0601213

Novello, M.; Perez Bergliatta, S. E.: Bouncing cosmology. Phys. Repts. 463, 127 (2008).

Riess, A. G. et al. [Supernova Search Team Collaboration]: Observation evidence from super-novae for an accelerating universe and a cosmological constant. Astron. J. 116, 1009 (1998), astro-ph/9805201

Perlmutter, S. et al. [SNCP Collaboration]: Measurements of omega and lambda from 42 high-redshift supernovae. Astrophys. J. 517, 565 (1999), astro-ph/9812133

Timoshkin, A. V.: Specially coupled dark energy in the oscillating FRW cosmology. J. Open Astronomy 2, 39 (2009).

Wei, H.; Wang, L. F.; Guo, X. J.: Quasi-rip: a new type of rip model without cosmic dooms-day. Phys.Rev. D 86, 083003 (2012), arXiv:1207.2898,v1 [gr-qc].

This manuscript was prepared with the AAS IATEX macros v5.2. 